\title{
Combining anatomy and physiology: New angiography-based and computed tomography coronary angiography-derived fractional flow reserve indices
}

\author{
Mariusz Tomaniak ${ }^{1}$, Patrick W. Serruys ${ }^{2,3}$ \\ ${ }^{1}$ First Department of Cardiology, Medical University of Warsaw, Poland \\ ${ }^{2}$ National Heart and Lung Institute, Imperial College London, United Kingdom \\ ${ }^{3}$ Department of Cardiology, National University of Ireland, Galway, Ireland
}

The first reports on deriving pressure loss from anatomy by simulating coronary flow date back 40 years [1]. The pursuit of anatomy-based coronary physiology assessment and its clinical translation has since been accelerated by Paul Morris et al. (2013) and Shengxian Tu et al. (2014)

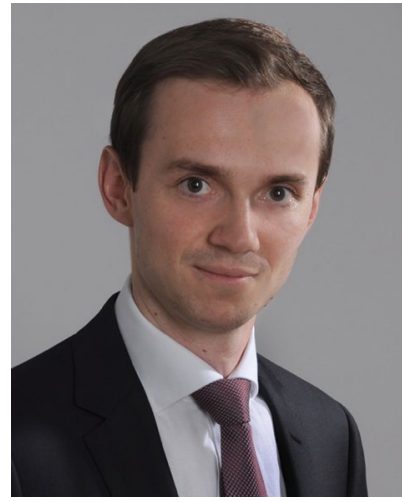
who computed fractional flow reserve (FFR) by applying computational fluid dynamics (CFD) simulation to three-dimensional (3D) coronary artery geometries extracted from two angiographic projections [2-4], and by Michail Papafaklis et al. (2014) who developed the virtual functional assessment index (vFAI) to predict flow-limiting coronary stenosis [5].

Avoidance of a pressure wire or microcatheter insertion into the coronary tree, lowers cost, procedural time and patient discomfort - in cases where a hyperemic agent is used - well justify the development of new software for wire-free $3 \mathrm{D}$ quantitative coronary angiography (QCA)-based FFR estimation, and the efforts being made to bring such modalities into clinical practice. At present, three validated vendor specific technologies have the promise to substantially improve the clinical adoption of physiological coronary lesion assessment in the routine practice of catheterization

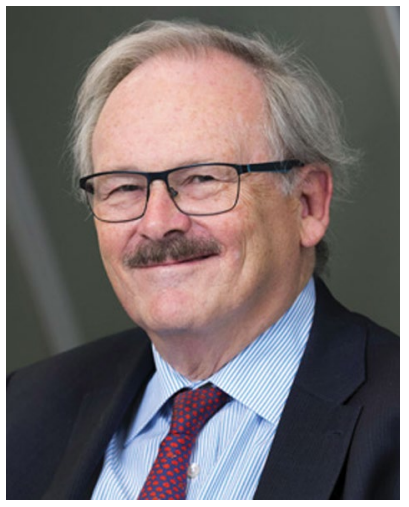

laboratory: quantitative flow ratio (QFR, Angio XA 3D software, Medis Medical Imaging System bv, the Netherlands and AngioPlus, Pulse Medical Imaging Technology, Shanghai, China), vessel fractional flow reserve (vFFR, CAAS Workstation, Pie Medical Imaging, Maastricht, the Netherlands), and $\mathrm{FFR}_{\text {angio }}\left(\mathrm{FFR}_{\text {angio }}\right.$ system, CathWorks, Ltd, Kfar-Saba, Israel) [6-9]. Attempts of predicting pulsatile vascular physiology on the basis of steady flow assumptions in CFD analyses have resulted in the development of mathematical methods to accelerate computation of angiography-based FFR estimation [10]. Concurrently, computed tomography coronary angiography (CTCA)-based $\mathrm{FFR}\left(\mathrm{FFR}_{\mathrm{CT}}\right)$ has also shown high correlation with pressure wire-based FFR and a high accuracy in detecting ischemia causing lesions [11], avoiding both coronary instrumentation and invasive angiography (Fig. 1).

\section{D-QCA-derived FFR}

$\mathrm{QFR}$, vFFR and $\mathrm{FFR}_{\text {angio }}$ demonstrated a significantly higher diagnostic accuracy, compared with traditional two-dimensional- or 3D-QCA [7-9]. While the requirement of minimum two angio-

Address for correspondence: Prof. Patrick W. Serruys, MD, PhD, P.O. Box 2125, 3000 CC Rotterdam, the Netherlands, tel: +31-10-4635260, fax: +31-10-4369154, e-mail: patrick.w.j.c.serruys@gmail.com 


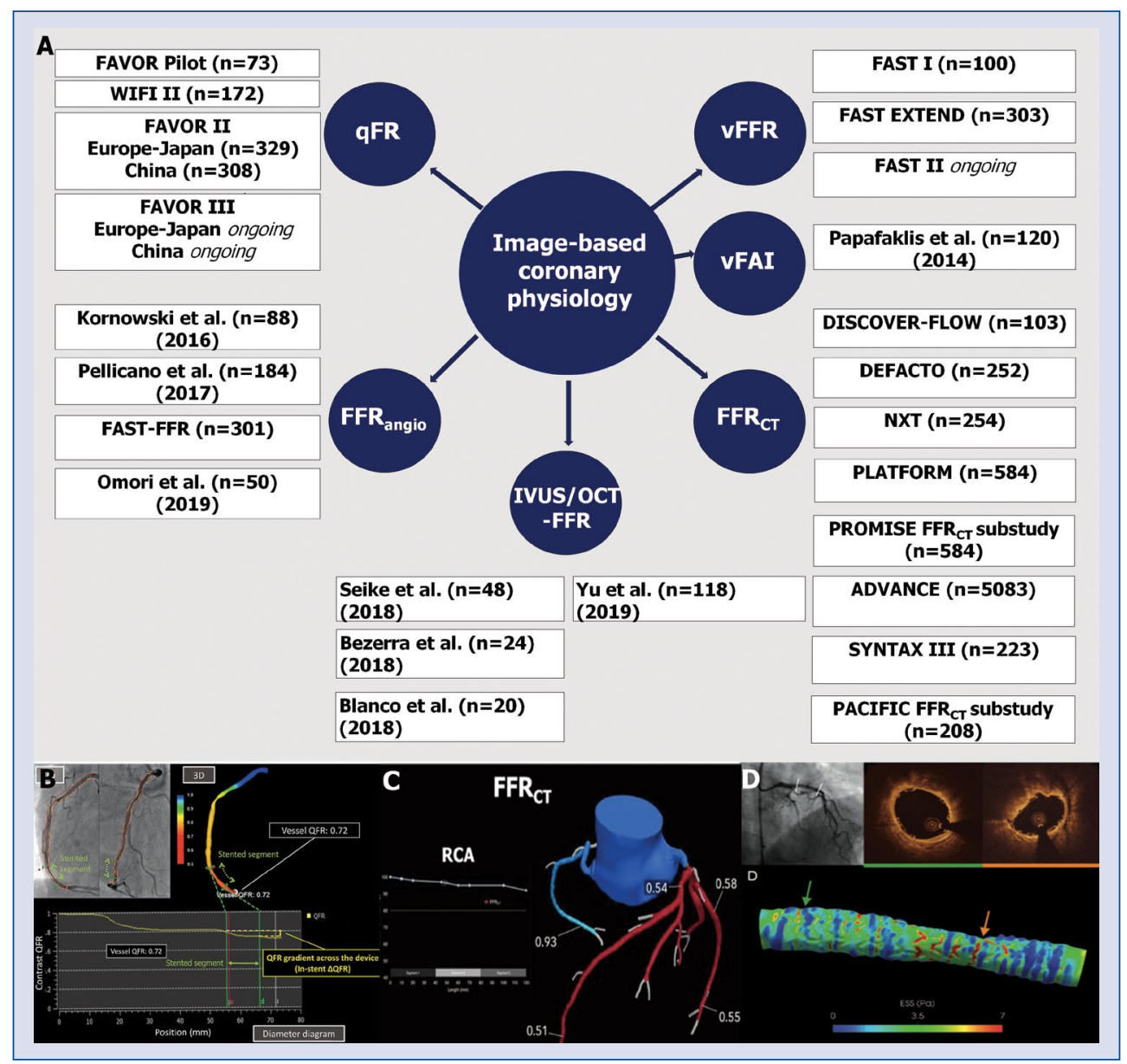

Figure 1. Imaging-based coronary physiology assessment; A. Graphic summary of imaging-based modalities for functional evaluation of coronary stenosis; B, C. Examples of quantitative coronary angiography- and computed coronary tomography angiography-based fractional flow reserve estimation; D. Computational fluid dynamics for wall shear stress computation; qFR - quantitative flow ratio; vFFR - vessel fractional flow reserve; $F_{F R}$ angio - fractional flow reserve derived from angiography; vFAI — virtual functional assessment index; FFR — fractional flow reserve; IVUS — intravascular ultrasound; OCT — optical coherence tomography; RCA — right coronary artery. Adapted with permissions from: Asano T et al. Eurolntervention. 2018; 14: 570-579; Modolo R et al. Ann Cardiothorac Surg. 2018; 7: 470-482; Thondapu V et al. Eur Heart J. 2018; 7: 1602-1609.

graphic projections with views of at least $25^{\circ}$ apart, brisk contrast injection and possibly minimized vessel overlap resulted in non-negligible exclusion rates in retrospectively analyzed cohorts to date $[8,12,13]$, the feasibility of angiography-based FFR computation was relatively high in prospectively enrolled cohorts with optimized protocols for angiography acquisition $[7,8,14]$. Of note, none of the three technologies has demonstrated the safety or non-inferiority of angio-based FFR versus the pressure-wire based FFR/iFR with regard to impact on clinical endpoints to date. However, large prospective studies are ongoing with specific focus on clinical follow-up and prespecified angiography acquisition protocols: FAVOR III China
(ClinicalTrial.gov: NCT03656848) and FAVOR III Europe-Japan (ClinicalTrials.gov: NCT03729739) will reveal whether QFR-guided revascularization may improve outcomes of patients undergoing percutaneous coronary intervention (PCI), as compared to subjects treated, respectively, based solely on angiography or angiography and pressure-wire based FFR (Fig. 1).

\section{PCI optimization: IVUS-FFR, OCT-FFR and post-PCI 3D-QCA-FFR}

Another promising multimodality approach to imaging of the coronary artery is derived from $3 \mathrm{D}$ artery models of angiography and grey-scale 
intravascular ultrasound (IVUS) - a concept initially proposed in 2000 by Slager et al. [15], and more recently pursued by the groups of Seike and Bezerra, amongst others, who reported a correlation between IVUS-derived FFR and pressure-wire based FFR, with an area under the curve reaching 0.93. In addition, optical coherent tomography (OCT) can be utilized for FFR estimation (OCT-derived FFR [OFR]), with a high diagnostic accuracy, as compared with conventional FFR values $[16,17]$. In a recent study by Huang et al. [17] in unselected patients with coronary syndrome, OFR was found superior to QFR in determining physiological significance of coronary stenosis and its diagnostic performance was not influenced by the presence of prior myocardial infarction or implanted stents. Both IVUS- and OCT-based FFR indices could serve as an additional means of final PCI result optimization, in particular when the procedure is already being guided with either of two imaging modalities. One of the major hurdles in reliable IVUS-FFR estimation related to stenosis length - subject to considerable inter-observer variability as reported in some prior studies - has been recently addressed by Kashiyama et al., who showed that stenosis length determined based on the area stenosis, rather than plaque burden, provides higher diagnostic accuracy of IVUS-FFR for physiologic ischemia detection (presented at the American Heart Association 2019 Conference, Philadelphia, US). However, clinical efficacy of intravascular imaging-based indices still remains to be confirmed in larger studies powered to evaluate clinical outcomes.

Interestingly, 3D-QCA-based functional indices computed using the angiograms acquired directly post PCI proved useful for stratification of risk after a successful procedure [18], including patients treated for de novo 3-vessel disease [19]; risk of vessel-oriented composite endpoint (vessel-related cardiac death, vessel-related myocardial infarction, and target vessel revascularization) was found to be 3-fold higher when post-PCI QFR was $\leq 0.89$ [18] or $\leq 0.90$ [19]. Consistent observations were also reported at the Transcatheter Cardiovascular Therapeutics 2019 (TCT 2019) for post PCI vFFR, with vessels presenting post-PCI vFFR values $>0.9$ having lower risk of target vessel revascularization at 1 year, post procedure, as compared to vessels with post PCI vFFR $\leq 0.9$ ( $1.8 \%$ vs. $4.2 \%, \mathrm{p}<0.05)$ (Masdjedi et al. presented at TCT 2019).

\section{CTCA-based FFR $\left(\right.$ FFR $\left._{\mathrm{CT}}\right)$}

Assessment of functional lesion severity based on CFD extends the CTCA capacities for lumen obstruction and plaque characteristics evaluation [11, $20,21]$. Recently, $\mathrm{FFR}_{\mathrm{CT}}$ has demonstrated similar ability to predict invasive FFR values as classic single photon emission computed tomography, being, however, inferior to cardiac positron emission tomography (Fig. 1) [22]. It has also proved safe with deferring lesions with $\mathrm{FFR}_{\mathrm{CT}}$ values above 0.8 , and could efficiently guide revascularization strategy with coronary artery bypass grafting or PCI, as was shown in the prospective SYNTAX III Revolution trial $[20,23]$. While the role of $\mathrm{FFR}_{\mathrm{CT}}$ in patient screening, detailed assessment of coronary lesion complexity and procedural planning is increasingly recognized, relatively long computation times, costs and a need for telemedicine have to be considered. Although in the United Kingdom the HeartFlow $\mathrm{FFR}_{\mathrm{CT}}$ analysis has been selected for reimbursement as part of the Innovation and Technology Payment (ITP) program, lack of reimbursement in majority of the countries nowadays represents non-negligible obstacle in rendering this technology more widely and clinically adoptable.

\section{Future directions}

In the recent ISCHEMIA (International Study of Comparative Health Effectiveness With Medical and Invasive Approaches) trial - presented by Judith S. Hochman at the American Heart Association Annual Scientific Sessions (AHA 2019) - routine invasive therapy failed to reduce major adverse ischemic events over a median of 3.3 years, compared with optimal medical therapy among chronic coronary syndrome patients with moderate to severe ischemia on noninvasive stress testing. There was also no benefit from invasive therapy regarding all-cause mortality or cardiovascular mortality/myocardial infarction. As such, additional diagnostic measures including more 'subtle' parameters such as frictional force exerted on the vessel wall by circulating blood, namely wall shear stress (WSS) - that currently can also be estimated in vivo based on either cine-angiography or CTCA scans - may prove efficient in improving risk stratification in the near future. It is conceivable that imaging modalities enriched with WSS information could facilitate appropriate identification of patients with signs of ischemia by traditional non-invasive 
tests, in whom interventional treatment could prevent hard clinical endpoints beyond relieving the angina symptoms. Indeed, the CFD sub analysis of the FAME II (Fractional Flow Reserve Versus Angiography for Multivessel Evaluation II) trial showed, that among patients with chronic coronary syndromes and hemodynamically significant lesions, higher WSS in the proximal segments of atherosclerotic lesions was predictive of myocardial infarction and had incremental prognostic value over FFR [24]. Recent standardization of WSS metrics and computation protocols [25] paves the way for further enhancing the current state-of-the-art of functional lesion assessment, potentially optimizing treatment decisions and improving the results of physiology-based coronary revascularizations in the 'post ICHEMIA trial' era. Finally, it has to be noted that both angio- and CTCA-derived FFR indices are restricted to epicardial arteries and imply a maximal relaxation of the vascular tone. Therefore, in future, comprehensive multimodality diagnostics combining anatomic and physiologic approaches will also need to better account for the vasomotion and coronary microvasculature assessment.

Conflict of interest: Mariusz Tomaniak acknowledges funding received as the Laureatte of the European Society of Cardiology Research and Training Program in the form of the 2018 ESC Grant. Patrick W. Serruys reported personal fees from Abbott, Biosensors, Medtronic, Micell, Sinomed, Philips/ /Volcano, Xeltis and HeartFlow, outside the submitted work.

\section{References}

1. Brown BG, Bolson E, Frimer M, et al. Quantitative coronary arteriography: estimation of dimensions, hemodynamic resistance, and atheroma mass of coronary artery lesions using the arteriogram and digital computation. Circulation. 1977; 55(2): 329-337, doi: 10.1161/01.cir.55.2.329, indexed in Pubmed: 832350.

2. Serruys PW, Girasis C, Papadopoulou SL, et al. Non-invasive fractional flow reserve: scientific basis, methods and perspectives. EuroIntervention. 2012; 8(4): 511-519, doi: 10.4244/EIJV8I4A79, indexed in Pubmed: 22581414.

3. Tu S, Barbato E, Köszegi Z, et al. Fractional flow reserve calculation from 3-dimensional quantitative coronary angiography and TIMI frame count: a fast computer model to quantify the functional significance of moderately obstructed coronary arteries. JACC Cardiovasc Interv. 2014; 7(7): 768-777, doi: 10.1016/j. jcin.2014.03.004, indexed in Pubmed: 25060020.

4. Morris PD, Ryan D, Morton AC, et al. Virtual fractional flow reserve from coronary angiography: modeling the significance of coronary lesions: results from the VIRTU-1 (VIRTUal Fractional Flow Reserve From Coronary Angiography) study. JACC Cardiovasc Interv. 2013; 6(2): 149-157, doi: 10.1016/j.jcin.2012.08.024, indexed in Pubmed: 23428006.
5. Papafaklis MI, Muramatsu T, Ishibashi Y, et al. Fast virtual functional assessment of intermediate coronary lesions using routine angiographic data and blood flow simulation in humans: comparison with pressure wire - fractional flow reserve. EuroIntervention. 2014; 10(5): 574-583, doi: 10.4244/EIJY14M07_01, indexed in Pubmed: 24988003.

6. Collet C, Onuma Y, Sonck J, et al. Diagnostic performance of angiography-derived fractional flow reserve: a systematic review and Bayesian meta-analysis. Eur Heart J. 2018; 39(35): 3314-3321, doi: 10.1093/eurheartj/ehy445, indexed in Pubmed: 30137305.

7. Westra J, Andersen BK, Campo G, et al. Diagnostic performance of in-procedure angiography-derived quantitative flow reserve compared to pressure-derived fractional flow reserve: the FAVOR II Europe-Japan study. J Am Heart Assoc. 2018; 7(14), doi: 10.1161/JAHA.118.009603, indexed in Pubmed: 29980523.

8. Masdjedi K, van Zandvoort LJC, Balbi MM, et al. Validation of 3-Dimensional Quantitative Coronary Angiography based software to calculate Fractional Flow Reserve: Fast Assessment of STenosis severity (FAST)-study. EuroIntervention. 2019, doi: 10.4244/EIJ-D-19-00466, indexed in Pubmed: 31085504.

9. Fearon WF, Achenbach S, Engstrom T, et al. FAST-FFR Study Investigators. Accuracy of Fractional Flow Reserve Derived From Coronary Angiography. Circulation. 2019; 139(4): 477-484, doi: 10.1161/CIRCULATIONAHA.118.037350, indexed in Pubmed: 30586699.

10. Morris PD, Silva Soto DA, Feher JFA, et al. Fast Virtual Fractional Flow Reserve Based Upon Steady-State Computational Fluid Dynamics Analysis: Results From the VIRTU-Fast Study. JACC Basic Transl Sci. 2017; 2(4): 434-446, doi: 10.1016/j. jacbts.2017.04.003, indexed in Pubmed: 28920099.

11. Koo BK, Erglis A, Doh JH, et al. Diagnosis of ischemia-causing coronary stenoses by noninvasive fractional flow reserve computed from coronary computed tomographic angiograms. Results from the prospective multicenter DISCOVER-FLOW (Diagnosis of Ischemia-Causing Stenoses Obtained Via Noninvasive Fractional Flow Reserve) study. J Am Coll Cardiol. 2011; 58(19): 1989-1997, doi: 10.1016/j.jacc.2011.06.066, indexed in Pubmed: 22032711.

12. Buono A, Mühlenhaus A, Schäfer T, et al. QFR predicts the incidence of long-term adverse events in patients with suspected CAD: feasibility and reproducibility of the method. J Clin Med. 2020; 9(1), doi: 10.3390/jcm9010220, indexed in Pubmed: 31947542 .

13. Kołtowski Ł, Zaleska M, Maksym J, et al. Quantitative flow ratio derived from diagnostic coronary angiography in assessment of patients with intermediate coronary stenosis: a wire-free fractional flow reserve study. Clin Res Cardiol. 2018; 107(9): 858-867, doi: 10.1007/s00392-018-1258-7, indexed in Pubmed: 30128817.

14. Xu Bo, Tu S, Qiao S, et al. Diagnostic accuracy of angiographybased quantitative flow ratio measurements for online assessment of coronary stenosis. J Am Coll Cardiol. 2017; 70(25): 3077-3087, doi: 10.1016/j.jacc.2017.10.035, indexed in Pubmed: 29101020 .

15. Slager CJ, Wentzel JJ, Schuurbiers JC, et al. True 3-dimensional reconstruction of coronary arteries in patients by fusion of angiography and IVUS (ANGUS) and its quantitative validation. Circulation. 2000; 102(5): 511-516, doi: 10.1161/01.cir.102.5.511, indexed in Pubmed: 10920062.

16. Yu W, Huang J, Jia D, et al. Diagnostic accuracy of intracoronary optical coherence tomography-derived fractional flow reserve 
for assessment of coronary stenosis severity. EuroIntervention. 2019; 15(2): 189-197, doi: 10.4244/EIJ-D-19-00182, indexed in Pubmed: 31147309.

17. Huang J, Emori H, Ding D, et al. Comparison of diagnostic performance of intracoronary optical coherence tomography-based and angiography-based fractional flow reserve for evaluation of coronary stenosis. EuroIntervention. 2020 [Epub ahead of print], doi: 10.4244/EIJ-D-19-01034, indexed in Pubmed: 31951207.

18. Biscaglia S, Tebaldi M, Brugaletta S, et al. Prognostic value of QFR measured immediately after successful stent implantation: the international multicenter prospective HAWKEYE study. JACC Cardiovasc Interv. 2019; 12(20): 2079-2088, doi: 10.1016/j. jcin.2019.06.003, indexed in Pubmed: 31563688.

19. Kogame N, Takahashi K, Tomaniak M, et al. Clinical implication of quantitative flow ratio after percutaneous coronary intervention for 3-vessel disease. JACC Cardiovasc Interv. 2019; 12(20): 2064-2075, doi: 10.1016/j.jcin.2019.08.009, indexed in Pubmed: 31563682

20. Fairbairn TA, Nieman K, Akasaka T, et al. Real-world clinical utility and impact on clinical decision-making of coronary computed tomography angiography-derived fractional flow reserve: lessons from the ADVANCE Registry. Eur Heart J. 2018; 39(41): 3701-3711, doi: 10.1093/eurheartj/ehy530, indexed in Pubmed: 30165613.
21. Collet C, Miyazaki Y, Ryan N, et al. Fractional flow reserve derived from computed tomographic angiography in patients with multivessel CAD. J Am Coll Cardiol. 2018; 71(24): 2756-2769, doi: 10.1016/j.jacc.2018.02.053, indexed in Pubmed: 29802016.

22. Driessen RS, Danad I, Stuijfzand WJ, et al. Comparison of coronary computed tomography angiography, fractional flow reserve, and perfusion imaging for ischemia diagnosis. J Am Coll Cardiol. 2019; 73(2): 161-173, doi: 10.1016/j.jacc.2018.10.056, indexed in Pubmed: 30654888.

23. Andreini D, Modolo R, Katagiri Y, et al.SYNTAX III REVOLUTION Investigators. Coronary computed tomography angiography for heart team decision-making in multivessel coronary artery disease. Eur Heart J. 2018; 39(41): 3689-3698, doi: 10.1093/ eurheartj/ehy581, indexed in Pubmed: 30312411.

24. Kumar A, Thompson EW, Lefieux A, et al. High coronary shear stress in patients with coronary artery disease predicts myocardial infarction. J Am Coll Cardiol. 2018; 72(16): 1926-1935, doi: 10.1016/j.jacc.2018.07.075, indexed in Pubmed: 30309470.

25. Gijsen F, Katagiri Y, Barlis P, et al. Expert recommendations on the assessment of wall shear stress in human coronary arteries: existing methodologies, technical considerations, and clinical applications. Eur Heart J. 2019; 40(41): 3421-3433, doi: 10.1093/ eurheartj/ehz551, indexed in Pubmed: 31566246. 